\title{
The CRAN bayesvl package on Renjin
}

\author{
Renjin \\ December 5, 2019

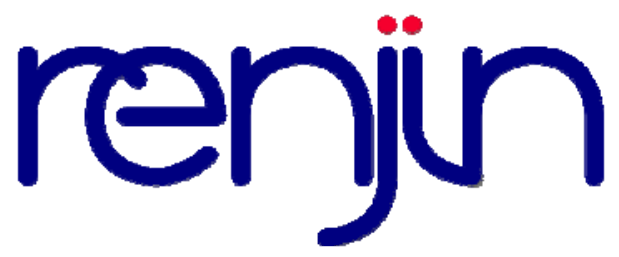

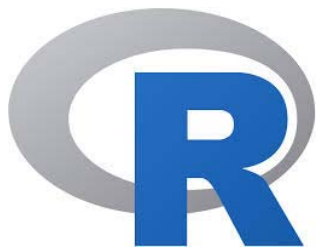

The R Core Team
The JVM-based interpreter for the R language

http://packages.renjin.org/package/org.renjin.cran/bayesvl 
Home

Resources

Support

Blog

\section{renjin}

CRAN

\section{bayesvl 0.8.5}

Visually Learning the Graphical Structure of Bayesian

Networks and Performing MCMC with 'Stan'

Released May 24, 2019 by Viet-Phuong La

This package cannot yet be used with Renjin it depends on other packages which are not available: rstan 2.18.2 and bayesplot 1.7.0

\section{Dependencies}

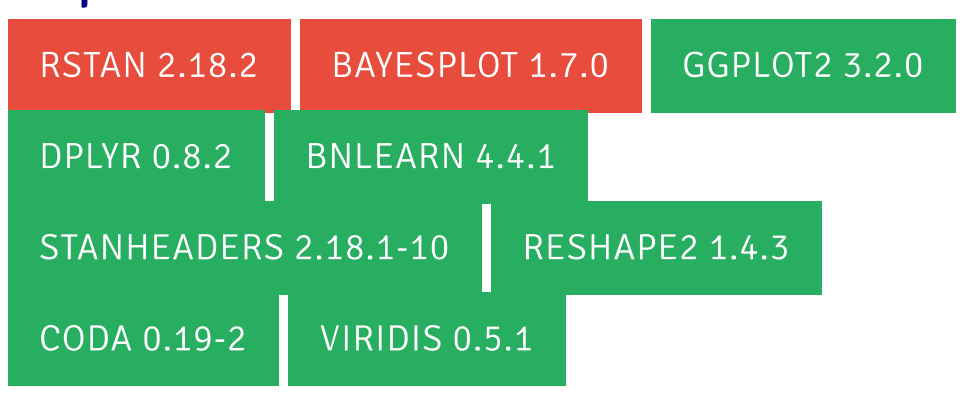

Provides users with its associated functions for pedagogical purposes in visually learning Bayesian networks and Markov chain Monte Carlo (MCMC) computations. It enables users to: a) Create and examine the (starting) graphical structure of Bayesian networks; b) Create random Bayesian networks using a dataset with customized constraints; c) Generate
Source

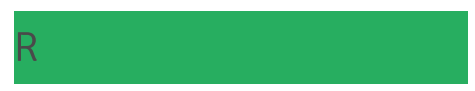

View GitHub Mirror

\section{Release History}

»0.8.5 
'Stan' code for structures of Bayesian networks for sampling the data and learning parameters; d) Plot the network graphs; e) Perform Markov chain Monte Carlo computations and produce graphs for posteriors checks. The package refers to one reference item, which describes the methods and algorithms: Vuong, Quan-Hoang and La, Viet-Phuong (2019) The 'bayesvl' $\mathrm{R}$ package. Open Science Framework (May 18).

\section{Downloads}

Take a look at our download page, or choose one of our downloads directly.

» Renjin CLI for Debian/Ubuntu [.deb]

» Renjin Studio (GUI) for all platforms [.jar]

» Renjin Script Engine for Java projects [.jar]

\section{Resources}

» Documentation

»Case studies

\section{Contribute}

Renjin is an open-source project. Go to the source code on GitHub.

\section{Support}

» Renjin Enterprise Support

»StackOverflow 


\section{References}

La, V. P., \& Vuong, Q. H. (2019). bayesvl: Visually learning the graphical structure of Bayesian networks and performing MCMC with 'Stan'. The Comprehensive R Archive Network (CRAN). Available online: https://cran.r-project.org/package=bayesvl

Vuong, Q. H., \& La, V. P. (2019). BayesVL package for Bayesian statistical analyses in R. GitHub. Available online: https://github.com/sshpa/bayesvl

Vuong, Q. H., La, V. P., Nguyen, M. H., Ho, M. T., Ho, M. T., \& Mantello, P. (2020). Improving Bayesian statistics understanding in the age of Big Data with the bayesvl R package. Software Impacts, 4, 100016.

Vuong, Q. H., La, V. P., Nguyen, M. H., Ho, M. T., Tran, T., \& Ho, M. T. (2020). Bayesian analysis for social data: A step-by-step protocol and interpretation. MethodsX, 7, 100924. 